\title{
A closer look at manifest consequence ${ }^{\dagger}$
}

\author{
Max Weiss \\ University of British Columbia
}

\begin{abstract}
Kit Fine (2007) argues that Frege's puzzle and its relatives demonstrate a need for a basic reorientation of the field of semantics. According to this reorientation, the domain of semantic facts would be closed not under the classical consequence relation but only under a stronger relation Fine calls "manifest consequence." I examine Fine's informally sketched analyses of manifest consequence, showing that each can be amended to determine a class of strong consequence relations. A best candidate relation emerges from each of the two classes, and I prove that the two candidates extensionally coincide. The resulting consequence relation is of independent interest, for it might be held to constitute a cogent standard of reasoning that proceeds under a deficient grasp on the identity of objects.
\end{abstract}

\section{Introduction}

In his 2007 book Semantic Relationism, Kit Fine argues that Frege's puzzle and its relatives demonstrate a need for a basic reorientation of the field of semantics, a kind of Copernican revolution according to which the domain of semantics would become one of information rather than of facts. The truths of semantics would become epistemic requirements for the understanding of language. This demand for a reorientation of semantics derives from a proximate thesis that the semantic facts cannot be closed under logical consequence. For, Fine seems to have shown, we cannot insist on the logical closure of the semantic facts and at the same time maintain a certain distinction that according to Fine is the key to the solution of Frege's puzzle and its relatives.

Fine's approach to Frege's puzzle essentially reverses the traditional problematic, by seeking to explain not how an identity can be informative but rather how it can be trivial: how could the pure grasp of meaning guarantee some ability to discern the recurrence of objects? According to Fine, an obvious but mistaken answer is that an identity is trivial in virtue of a resemblance between the meanings of the two names in question. ${ }^{1}$ Conversely, an identity would be informative in virtue of a difference of meaning. But then the informativeness of identity statements gives rise to a proliferation of meanings for terms. To house the new diversity, the Fregean posits a second layer of meaning. On the other hand, the referentialist rejects the presupposition that informativeness must be explained semantically. ${ }^{2}$

Fine proposes to explain how the sentence "Cicero is Tully" may differ in meaning from the sentence "Cicero is Cicero", even though the meaning

$\dagger$ Forthcoming in JPL.

(C) 2013 Kluwer Academic Publishers. Printed in the Netherlands. 
of "Cicero" is the same as the meaning of "Tully". In this way, Fine plans to evade the difficulties faced by the referentialists on the one hand and the descriptivists on the other. However, it appears that Fine's proposal conflicts with the principle of compositionality. For if the meaning of "Cicero" is the meaning of "Tully", then a sentence whose meaning is a function of the meanings of "Cicero" and of "Tully" cannot differ in meaning from a sentence whose meaning is the very same function of the meanings of "Cicero" and "Cicero". Fine suggests ingeniously that we ought instead to regard the meaning of each sentence as a function not of the intrinsic meanings of the one name and of the other, but rather as a function of the meaning of the two names together. Perhaps a semantic relation holds between the two names that does not supervene on their intrinsic meanings in the first place. ${ }^{3}$ Terms which stand in such a relation would be said to corefer strictly; not only would they represent the same object but they would represent it "as the same". 4

Fine thinks of semantics as a science, and seems, in Aristotelian fashion, to think of a science as having a proper domain or subject-matter. The domain of a science consists of some totality of facts. In particular, the facts of semantics are those which hold purely in virtue of the meanings of expressions. This very broad picture leads Fine to propose, not so much as an analysis but as an informal characterization, that the coreference of two terms will be strict if it is no mere accident, but a genuine fact of semantics. ${ }^{5}$

To see how the proposal might apply to the case at hand, first consider a statement of identity whose flanking terms corefer strictly. Since it is part of the meaning of the terms together that they do corefer, a speaker cannot really understand the statement without recognizing its truth. Conversely, consider a true statement of identity which is genuinely informative: for example, the sentence "Cicero is Tully." Now, that "Cicero" refers to Cicero is a semantic fact, and that "Tully" refers to Cicero is a semantic fact as well. Does it follow that it is a semantic fact that "Cicero" and "Tully" corefer? Such a fact seems surely relevant to what is expressed by the sentence "Cicero is Tully". But then, without somehow refining the idea of facts as holding in virtue of a sentence's meaning what it does, we remain stuck with the unwanted conclusion that a full grasp of the meaning of a true identity puts a speaker in a position to acknowledge its truth.

Faced with this problem, Fine takes drastic recourse: he suggests that the domain of semantics, unlike the domain of an ordinary science, is not closed under logical consequence. In other words, what follows logically from what holds purely in virtue of the meanings of some expressions need not itself hold purely in virtue of those meanings. So in particular, facts of coreference need not hold purely in virtue of the meanings of individual names. Then, knowledge of the semantic facts would not require knowledge of the facts of coreference. 
Now, it might be that this very general speculation could somehow help with Frege's puzzle. But at what cost? If the domain of semantics were supposed to lack any logical structure at all, then the alleged science of semantics might seem to become a kind of poetry or storytelling which we cannot really appreciate without lowering our critical faculties. ${ }^{6}$ A second concern is that on Fine's view, in order to arrive at understanding of what is said, a speaker must apply general semantic facts to the particular utterance encountered. ${ }^{7}$ But it would be by means of such a process that a speaker's interpretation not only happens to emerge, but emerges with justification. It is, after all, possible to misunderstand what is said. Yet the interpretive process confers justification presumably because it is rational, and therefore proceeds by means of logical principles. So we cannot suppose the domain of semantics to be constituted independently of logic altogether.

Here is where Copernicus comes in. Perhaps we should not conceive of semantics as discovering independently constituted facts to which a speaker's mind must conform. Maybe instead the point is to describe the knowledge that explains the grasp of meaning of particular utterances. In this way, the primary question of semantics becomes what must subjectively have been given in order that linguistic understanding be possible. Now, semantic knowledge can be taken to explain understanding of an utterance only in virtue of inferences that it is reasonable to demand of a speaker. ${ }^{8}$ And in particular, it would be unreasonable to demand of a speaker a totally unblinkered capacity for recognition of objects as the same again.

Thus we are led in pursuit of some strengthening of the classical consequence relation, under which the domain of semantics, construed as a domain of requirements rather than of facts, may be said to be closed. The intended relation, which Fine labels "manifest consequence", will be one that legitimates exactly those inferences which a speaker can draw without needing to see through the variety of his takes on objects. ${ }^{9}$ For example, a speaker might be told "Cicero is an orator" and "Tully is Roman", and, granting the correctness of these assertions, so become privy to the facts that Cicero is an orator and that Cicero is Roman. But we cannot thereby take the speaker to be privy to the fact that someone is a Roman orator. Thus, existential generalization ought not always to be manifestly available. Similarly, we cannot expect a speaker always to be capable of recognizing instances of modus ponens, not in case some recurrence of an object in the hypotheses affects the instancing of this form. In contrast, however, disjunction introduction ought to be manifestly available, because, for example, a speaker who knows that Cicero is an orator can certainly infer that either Cicero is an orator, or Cicero is a spy. Since the speaker as it were "stipulates" that the Cicero of each disjunct of that conclusion be precisely the Cicero of the hypotheses, it may therefore also be open for the speaker to infer that somebody is either a Roman or a spy. 
In $S R$, which is based on a series of lectures, Fine sketches two analyses of the manifest consequence relationship. The first analysis develops the idea that an inference is manifestly sound provided that it can be drawn "regardless of the identity of the objects in the hypotheses". ${ }^{10}$ For the second analysis, Fine first tries to characterize those consequences which can be drawn under this or that regime of strict coreference, and then identifies the manifest consequences as those that can be drawn under every such regime. ${ }^{11}$ However, Fine's analyses are stated informally, and it turns out that the first analysis admits of serious counterexamples. Furthermore, even if the first analysis can be amended, it is unclear that it can be amended in such a way as to coincide extensionally with the second analysis. This points to a second concern. The philosophical importance of the concept of manifest consequence depends to some extent on there corresponding to it some reasonably natural extension. Classical first-order consequence deserves our attention partly because it is the point of convergence of a wide variety of proof-theoretic and semantic analyses. If, in contrast, it turns out that the extension of the manifest consequence relation depends tightly on matters of technical detail, so that seemingly innocent adjustments yield new extensions, then this would weaken claims of an epistemologically central role for the corresponding informal idea.

The primary goal of what follows is to investigate the notion of manifest consequence as Fine characterizes it in his book. From this perspective there are a couple of prior notions invoked by Fine's analyses that call for a little more work. First, Fine's account of manifest consequence invokes a consequence relation over so-called 'singular' or 'Russellian' propositions. But in the mainstream development of mathematical logic, the consequence relation is usually taken to relate not propositions but sentences or formulas. ${ }^{12}$ So, to stabilize the discussion, $\S 2$ of this paper presents a couple of reasonable candidates for a 'standard' consequence relation over propositions. Second, Fine's analyses of manifest consequence quantify metalinguistically not just objects and propositions, but also over occurrences of objects in propositions; so, $\S 2$ continues by formulating an appropriate notion of occurrence. In $\S 3$, we begin the real business of the paper by stating Fine's first analysis and posing the promised counterexamples. After amending Fine's analysis, we establish some basic facts about the resulting concept. In $\S 4$, we investigate the more general notion of strict coreference, which is underpinned semantically by the notion of a coordination scheme over a body of propositions. After explicating the notion of consequence relative to such schemes, we establish some more results, in particular that Fine's second analysis does coincide with the first. The paper concludes in $\S 5$ with reflections on the structure and application of semantic relationism. 


\section{Framework}

The standard Tarskian approach to the semantics of first-order languages introduces the notions of structure and of satisfaction of a formula by a structure, perhaps relative to an assignment. Because, on this approach, a structure maps a closed formula directly to a truth-value, a notion of the content of a closed formula does not clearly arise. One might suppose that the definition of satisfaction induces a notion of content as an associated function from structures to truth-values, but since the domain of a structure is an arbitrary set, such a function will be a proper class. ${ }^{13}$ Thus in particular we cannot quantify over such contents. ${ }^{14}$

The semantic notion of a modal frame provides a way around the difficulty by providing a distinguished set of structures or "points" of evaluation. The meanings of formulas can then be identified with functions from elements of this set to truth-values. ${ }^{15}$ However, as is well known, this analysis assigns the same content to all formulas that are true at the same points. So, if truth-ata-point corresponds to logical satisfaction, then logically equivalent formulas would have the same content. It might then be suspected that no such resulting notion of content will be as fine-grained as the contents of ordinary sentences. ${ }^{16}$

An alternative conception reverts to the primal ideas of Russell and Moore, according to which the meaning of a sentence is a complex entity whose parts correspond to the meanings of the constituent words. ${ }^{17}$ David Kaplan revived this approach as an heuristic for an account of the semantics of directly referential expressions, in particular of indexicals. ${ }^{18}$ According to Kaplan's account, semantic evaluation falls into two stages. Relative to some background structure, we first consider a "context of utterance", which maps a sentence to a propositional content. Kaplan urges us to think of propositional content as built up from the values of the referring constituents of the sentence. Thus, for example, there is a context in which the sentence "I am not David Kaplan" expresses a proposition containing David Kaplan and Joseph Almog, together, perhaps, with the identity relation and a negation operator. Now at the second stage of evaluation we identify within the background structure a so-called "circumstance of evaluation", and check whether the Russellian proposition holds of the circumstance. In our running example, this amounts to checking whether ' $x \neq y$ ' is true in the structure relative to the assignment of Almog to ' $x$ ' and Kaplan to ' $y$ '-in other words, to checking whether Almog is Kaplan. ${ }^{19}$

\subsection{SyNTAX, TRUTH AND CONSEQUENCE FOR $\mathcal{L}_{\mathcal{U}}$}

We will work against the background of a first-order language $\mathcal{L}_{\emptyset}$ all of whose nonlogical expressions are predicates. The closed formulas of $\mathcal{L}_{0}$ are to be 
understood as Russellian propositions, and formulas more generally may be understood as 0 -ary propositional functions. To this end, the predicates of $\mathcal{L}_{0}$ might be identified with properties and relations of the appropriate arity. Of course, Russellian propositions are famous for containing ordinary and extraordinary objects, such as Mont Blanc. So, let $\mathcal{U}$ be an infinite set of objects that is to be the source of constituents of Russellian propositions. We then take $\mathcal{L}_{\mathfrak{U}}$ be the language that results from $\mathcal{L}_{0}$ by adding to its nonlogical vocabulary the elements of $\mathcal{U}$ as constants. Thus, a formula of $\mathcal{L}_{\mathfrak{U}}$ can be understood as a propositional function over the source $\mathcal{U}$ of constituents, relative to the properties primitive to $\mathcal{L}_{\emptyset}$. Likewise, a proposition over $\mathcal{U}$ is a closed formula of $\mathcal{L}_{\mathfrak{U} l}$. More generally, it will be convenient at various stages to extend a language $\mathcal{L}_{X}$ whose constants are the elements of $X$, to a language $L_{X \cup Y}$ whose constants are the elements of $Y .{ }^{20}$

The theoretical role of Russellian propositions in semantics assigns them a kind of intermediate position between closed formula and truth-value. Our initial treatment of the expressions of $\mathcal{L}_{\mathcal{U}}$ will reflect this position. In particular, the terms of $\mathcal{L}_{\mathcal{U}}$ do not need to be assigned denotations, for they are denotations. Moreover, the predicates of $\mathcal{L}_{\mathfrak{U}}$ are really to be understood as properties or relations, but a structure is needed to determine which are the tuples that instantiate them. The task now becomes simply to define an appropriate notion of structure, and then to define the notion of truth relative to such structures.

A structure $\mathcal{M}$ for $\mathcal{L}_{\mathcal{U}}$ consists of a domain $|\mathcal{M}|$, together with a function that assigns each $k$-ary $\mathcal{L}_{\mathcal{U}}$-predicate $R$ a $k$-tuple $R^{\mathcal{M}}$ of elements of $|\mathcal{M}| \cup \mathcal{U}$. We run the inductive definition of truth-in- $\mathcal{M}$ on propositions directly, but on the propositions of the expanded language $\mathcal{L}_{|\mathcal{M}| \cup \mathcal{U}}$.

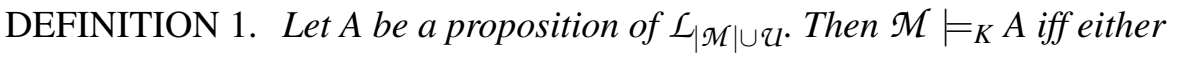

- $A$ is $\left\ulcorner R a_{1} \ldots a_{k}\right\urcorner$ and $\left\langle a_{1}, \ldots, a_{k}\right\rangle \in R^{\mathcal{M}}$, or

- $A$ is $\ulcorner a=b\urcorner$ and $a=b$,

- $A$ is $\ulcorner\neg B\urcorner$ and $\mathcal{M} \not \nvdash_{K} B$, or

- $A$ is $\ulcorner B \rightarrow C\urcorner$ and either $\mathcal{M} \not \models_{K} B$ or $\mathcal{M} \models_{K} C$, or

- $A$ is $\ulcorner\exists x B\urcorner$, and $\mathcal{M} \mid={ }_{K} B[a]$ for some $a \in|\mathcal{M}|$.

Using this definition we identify a consequence relation $\Rightarrow_{K}$ for Russellian propositions.

DEFINITION 2. Let $X$ be a sequence of $\mathcal{L}_{\mathcal{U}}$-propositions and let $A$ be an $L_{\mathcal{U}^{-}}$ proposition. Then $X \Rightarrow_{K}$ A iff for every $\mathcal{L}_{\mathfrak{U}}$-structure $\mathcal{M}$ we have $\mathcal{M} \models_{K} A$ unless $\mathcal{M} \forall \nvdash_{K} B$ for some proposition $B$ in $X$. 
While Definition 1 has an attractive simplicity, there are alternative approaches to evaluation of $\mathcal{L}_{\mathfrak{U}}$. For example, we might consider propositions not as semantic values but rather as exotically constituted expressions, treating the objectual propositional constituents no longer as the designations of terms but rather as terms themselves.In any case, under the reconstrual, $\mathcal{L}_{\mathfrak{U}}$ would resemble an ordinary first-order language. This alternative conception will play a crucial role in what follows, so we define it explicitly.

An $L$-structure $\mathcal{M}$ for $\mathcal{L}_{\mathcal{S}}$ consists of a $K$-structure together with a mapping that assigns a value $a^{\mathcal{M}} \in|\mathcal{M}|$ for each term $a \in \mathcal{S}$. If $\mathcal{M}$ is an $L$-structure for $\mathcal{L}_{S}$, and if $\mathcal{S}^{\prime}$ is a a set disjoint from $\mathcal{S}$, then $\mathcal{M}^{\prime}$ is an expansion of $\mathcal{M}$ to $\mathcal{S}^{\prime}$ provided that $\mathcal{M}^{\prime}$ is an $L$-structure for $\mathcal{L}_{\mathcal{S} \cup S^{\prime}}$ that coincides with $\mathcal{M}$ as an interpretation of $\mathcal{L}_{\mathcal{S}}$. An $L$-satisfaction definition now results from the $K$ satisfaction definition by exchanging ' $K$ ' for ' $L$ ' and replacing the atomic and quantificational clauses in the $K$-definition with the following:

- $A$ is $\left\ulcorner R a_{1} \ldots a_{k}\right\urcorner$ and $\left\langle a_{1}^{\mathcal{M}}, \ldots, a_{k}^{\mathcal{M}}\right\rangle \in R^{\mathcal{M}}$ for $R$ nonlogical, or

- $A$ is $\ulcorner a=b\urcorner$ and $a^{\mathcal{M}}=b^{\mathcal{M}}$,

- $A$ is $\ulcorner\exists x B\urcorner$, and $\mathcal{M}^{\prime} \models_{L} B[c]$ for $c$ a new constant and $\mathcal{M}^{\prime}$ an expansion of $\mathcal{M}$ to $\{c\}$.

A corresponding consequence relation $\Rightarrow_{L}$ now results by replacing ' $K$ ' with ' $L$ ' in the definition of $\Rightarrow_{K}$.

Comparison brings out two special features of $\Rightarrow_{K}$. The first feature concerns identity. Let $A$ be a formula $\ulcorner a=b\urcorner$. If $a=b$, then $\mathcal{M}=_{K} A$ for any $\mathcal{M}$, so that $A$ is valid. Conversely if $a \neq b$, then $\mathcal{M} \not \nvdash_{K} A$, and hence $\neg A$ is valid. So every proposition of identity is either $K$-valid or $K$-contravalid. This contrasts with $L$-consequence, since $\ulcorner a=b\urcorner$ is $L$-valid or $L$-contravalid if and only if $a=b$. In particular, since a proposition $\ulcorner a \neq b\urcorner$ is $L$-satisfiable only if $a \neq b$, therefore no proposition $\ulcorner a \neq b\urcorner$ is $L$-valid. On the other hand, $\ulcorner a \neq b\urcorner$ is $K$-valid whenever it is satisfiable, that is, whenever $a \neq b$. Here, $\Rightarrow_{K}$ agrees with the behavior of the corresponding Kaplanian contents, as it does also with valuated sentences. ${ }^{21}$

The second point of departure concerns the quantifiers. Consider a formula $A=\ulcorner F x\urcorner$ of $\mathcal{L}_{\mathcal{U}}$ and a $K$-structure $M$ such that $a \in F^{\mathcal{M}}$ but $a \notin|\mathcal{M}|$. Clearly $\mathcal{M} \models \models_{K} A[a]$ but $\mathcal{M} \not \models_{K} \exists x A$. Thus, existential generalizations are not always $K$-entailed by their instances. Likewise, universal generalizations do not $K$ entail their instances either. Here $K$-satisfaction agrees with Kaplan's account of satisfaction for valuated sentences. ${ }^{22,23}$

Of course, the two differences between $\Rightarrow_{K}$ and $\Rightarrow_{L}$ are conceptually separable. On the one hand, we might restore existential generalization by considering in the definition of $K$-consequence only those $K$-structures $\mathcal{M}$ such that $\mathcal{U} \subseteq|\mathcal{M}|$. Conversely, we can break universal instantiation while 
maintaining the logical contingency of nontrivial identities by introducing a restriction on the range of the object-language quantifier, as specified in the $L$-satisfaction definition, to a subset of $|\mathcal{M}|$.

\subsection{OCCURRENCES}

As mentioned in $\S 1$, Fine proposes to identify those inferences which the knowledge of all relevant semantic facts should allow logically competent speaker to draw. Suppose, now, that the names $m$ and $n$ both refer to $a$. Then, the relevant semantic facts about the sentence " $m \neq n$ " include that it expresses the proposition $\ulcorner a=a\urcorner$. But, a logically competent speaker can grasp the meaning of " $m \neq n$ " without recognizing that it expresses a falsehood. Since full grasp of meaning of the sentence entails recognizing that the proposition it expresses is $\ulcorner a \neq a\urcorner$, the speaker must fail to recognize that $\ulcorner a \neq a\urcorner$ is false. But a logically competent speaker must recognize that a proposition is false if it entails absurdity. So, it must sometimes arise that a logically competent speaker grasps the proposition $\ulcorner a \neq a\urcorner$ but fails to recognize that it entails absurdity. The explanation, according to Fine, is that logical competence does not in general require the knowledge that one and the same object occurs on both sides of the $\neq$. Supposing that it were not one but two objects that occurred in the two positions, then absurdity would no longer follow. Logical competence only requires recognition of those consequences that are impervious to such redistribution of objects through the positions in a bunch of hypotheses. Then a consequence is said to be manifest if its recognition is actually required by logical competence. But does anything deserve the name "manifest consequence relation"? Before entering into the question in detail, it may help spell out the underlying concept of the positions in which objects occur in a possibly infinite sequence of hypotheses.

We will take a sequence $\sigma$ to be a mapping whose domain is an ordinal $|\sigma|$. In fact it will be convenient to identify $\sigma$ with its graph $\{\langle\alpha, \sigma(\alpha)\rangle: \alpha<$ $|\sigma|\}$. If $\sigma$ and $\tau$ are both sequences, then the concatenation $\sigma \frown \tau$ of $\tau$ to $\sigma$ is the set $\sigma \cup\{\langle|\sigma|+\alpha, \tau(\alpha)\rangle: \alpha<|\tau|\}$. If $f$ is a function whose domain includes the range of $\sigma$, then $f \circ \sigma$ is the composition of $f$ to $\sigma$, so that $f \circ \sigma=$ $\{\langle\alpha, f(\sigma(\alpha))\rangle: \alpha<|\sigma|\}$. Note that if $\sigma(\alpha)=\rho(\alpha)$ for all $\alpha<|\sigma|$, then there is exactly one $\tau$ such that $\sigma \frown \tau=\rho$.

We apply this familiar machinery to represent occurrences of terms in formulas. When $A$ is a formula that contains exactly $k=|\sigma|$ free variables, then and only then we will write $A \sigma$ for the result $A[\sigma(0), \ldots, \sigma(k-1)]$ of everywhere substituting $\sigma(i)$ for the lexicographically $i$ th free variable in $A$. Now, say that a formula $A$ is nice if $A$ is a formula of $\mathcal{L}_{0}$, and if there is no variable with more than one free occurrence in $A$. Here is the fundamental idea. 
DEFINITION 3. Let $A$ be a formula. The associated nice formula $\bar{A}$ of $A$ is the lexicographically earliest nice formula such that $\bar{A} \sigma=A$ for some sequence $\sigma$.

Note that $\sigma$ is uniquely determined from $A$ by the definition as well. Thus, for example, if $A=\ulcorner F b \wedge a=b\urcorner$, then $\bar{A}=\left\ulcorner F x_{0} \wedge x_{1}=x_{2}\right\urcorner$, and $\sigma=\langle b, a, b\rangle$. In this way, every formula is represented by exactly one pair $\langle\bar{A}, \sigma\rangle$.

We actually want to keep track of multiplicity of occurrences across arbitrary sequences of propositions. If $X$ is a sequence of propositions $\left\langle A_{\alpha}\right.$ : $\alpha<\beta\rangle$, then we write $\bar{X}$ for $\left\langle\bar{A}_{\alpha}: \alpha<\beta\right\rangle$. The wellordering of a sequence of propositions $X$ induces a wellordering on occurrences of free variables in formulas of $\bar{X}$ as follows: an occurrence of $x_{i}$ in $\bar{A}_{\alpha}$ precedes an occurrence of $x_{j}$ in $\bar{A}_{\beta}$ iff either $\alpha<\beta$ or $\alpha=\beta$ and $i<j$. When $\sigma$ has this order type, then we write $\bar{X} \sigma$ for the result of substituting each $\sigma(\alpha)$ for the $\alpha$ th occurrence of a free variable in $\bar{X}$. Here too there is just one sequence $\sigma$ such that $X=\bar{X} \sigma$.

As an illustration of these ideas, we may say that a consequence relation $\Rightarrow$ respects substitution when $\bar{X} \sigma \Rightarrow \bar{A} \tau$ guarantees that $\bar{X}(f \circ \sigma) \Rightarrow \bar{A}(f \circ$ $\tau)$, for any $f: \mathcal{U} \rightarrow \mathcal{U}$. It is a standard result that $\Rightarrow_{L}$ respects substitution. However, $\Rightarrow_{K}$ does not: take for example $X=\emptyset, A=\ulcorner 0 \neq 1\urcorner$, and $f$ such that $f(0)=f(1)$. We do still have a weaker property, namely that $\Rightarrow_{K}$ respects substitution for $f$ injective.

\section{The differentiation-based analysis}

We now turn to Fine's initial sketch of the manifest consequence relation. Informally, the motivation is to describe those consequences that can be drawn independently of any ability to recognize objects as they occur or recur in the hypotheses. So suppose we hold fixed the sequence of positions in which an object occurs, and consider an arbitrary redistribution of objects throughout those positions. Then, according to the idea of Fine's first sketch, only if it is impervious to such redistribution through the hypotheses should the soundness of an argument be manifest.

Let us consider the sketch in more detail. According to Fine, a proposition $A^{\prime}$ differentiates a proposition $A$ provided that $A^{\prime}$ is the result of "replacing distinct occurrences of the same object by distinct objects" (Fine, 48). The intention of this proposal seems to be that distinct occurrences of one object must everywhere give way to occurrences of distinct objects. The proposal naturally extends to sequences of propositions. Thus, a sequence $A_{1}^{\prime}, A_{2}^{\prime}, \ldots$ of propositions differentiates the sequence $A_{1}, A_{2}, \ldots$ provided that distinct occurrences of one object throughout the sequence everywhere give way to occurrences of distinct objects. Following the body of the text, we proceed to an initial sketch of the manifest consequence relation: 
The proposition $B$ is an $m$-consequence of the sequence $A_{1}, A_{2}, \ldots$ of propositions provided that for every differentiation $A_{1}^{\prime}, A_{2}^{\prime}, \ldots$ of $A_{1}, A_{2}, \ldots$, there's a differentiation $B^{\prime}$ of $B$ such that $B^{\prime}$ is a classical consequence of $A_{1}^{\prime}, A_{2}^{\prime}, \ldots{ }^{24}$

Fine appends to his statement of this analysis a endnote with three further suggestions. ${ }^{25}$ The second suggestion seems fairly natural and straightforward, namely that the notion of "classical consequence" mentioned in the definition of manifest consequence could be varied. It might, for example, be worth considering relevant-logical base relations here instead. However, in this paper I consider just the effects of using combinations of $\Rightarrow_{K}$ and $\Rightarrow_{L}$.

The first and third suggestions are more crucial for present purposes. The first is that we might strengthen the definition of manifest consequence, by requiring any object occurring in the differentiated conclusion to occur as well in one of the differentiated premises. I will return to this shortly, but for now let me just remark that the suggestion seems to raise potential pigeonhole difficulties, when combined with the requirement that distinct occurrences everywhere give way to distinct objects. For example, somehow the conclusion $\ulcorner F a \vee F a\urcorner$ ought to follow manifestly from the premise $\ulcorner F a\urcorner$; but a differentiation of the premise will not contain enough objects to include all the objects that occur in a proposition that injectively differentiates the conclusion. A natural remedy here is to follow Fine's third suggestion in the endnote: "we might allow some distinct occurrences of the same object to correspond to the same object under the differentiation." The pigeonhole issue just mentioned indicates that the third suggestion may yield some desirable flexibility.

\subsection{DIFFERENTIATION}

In $\S 2$, we found a way to represent an arbitrary proposition $A$ as the result $\bar{A} \sigma$ of applying a substitution $\sigma$ to an associated "nice" formula $\bar{A}$. Using this machinery, it is straightforward to reconstruct various possible concepts of differentiation. The weakest concept is as follows:

DEFINITION 4. A proposition $A^{\prime}$ differentiates a proposition A provided that $A^{\prime}=\bar{A} \sigma^{\prime}$ for some $\sigma^{\prime}$.

Similarly, a sequence $X^{\prime}$ of propositions differentiates a sequence $X$ provided that $X^{\prime}=\bar{X} \sigma^{\prime}$ for some $\sigma^{\prime}$.

There are several ways in which the notion can be strengthened. First, we might insist that the differentiation eliminate all multiplicity of occurrences of any single object; this amounts to the requirement that $\sigma^{\prime}$ be injective. Second, we might insist that differentiation be "conservative" in the sense that it changes only what it must. More precisely, let $\operatorname{inj}(\sigma)$ be the set of all pairs $\langle\alpha, \sigma(\alpha)\rangle$ such that for no $\beta \neq \alpha$ have we $\sigma(\beta)=\sigma(\alpha)$; then conservativeness of a differentiation $\sigma^{\prime}$ amounts to the requirement that $\sigma^{\prime} \supseteq \operatorname{inj}(\sigma)$. Probably, 
the intention of the text is that a differentiation be both injective and conservative; endless variations are conceivable. But the weaker concept simplifies the technical situation; moreover the extra constraints do not resolve certain difficulties that Fine's initial analysis confronts.

\subsection{FINE'S SKETCHES}

Let me begin by restating Fine's initial sketch:

A proposition $A$ is an $m$-consequence of the sequence $X$ of propositions provided that for every differentiation $X^{\prime}$ of $X$, there's a differentiation $A^{\prime}$ of $A$ such that $A^{\prime}$ is a classical consequence of $X^{\prime}$.

We have defined four concepts of differentiation and two concepts of consequence for propositions; this yields eight readings of the sketch. But there is a difficulty that pervades all the readings in one form or another. First suppose differentiation to be as in Definition 4. The counterexample is then as follows.

- Let $B=\ulcorner a=a\urcorner$, and let $A=\ulcorner a=b\urcorner$. Now, suppose that $B^{\prime}$ is a differentiation of $B$. Then $B^{\prime}=\ulcorner c=d\urcorner$ for some objects $c$ and $d$. But $\ulcorner c=d\urcorner$ is a differentiation of $A$. So $B m$-implies $A$.

We might now try to strengthen the $m$-consequence relation by insisting that differentiations be conservative. However, by introducing "dummy" occurrences, such a restriction can be defanged:

- Let $T_{a}$ and $T_{b}$ be logically valid propositions that contain a single occurrence of an object, namely $a$ and $b$ respectively. And let $B=\ulcorner a=a\urcorner$, and let $A=\left\ulcorner T_{a} \wedge T_{b} \wedge a=b\right\urcorner$. Now, suppose that $B^{\prime}$ is a differentiation of $B$, so that $B^{\prime}$ is $\ulcorner c=d\urcorner$ for some objects $c$ and $d$. Then for any objects $e$ and $f$, the proposition $A^{\prime}=\left\ulcorner T_{e} \wedge T_{f} \wedge c=d\right\urcorner$ is a conservative differentiation of $A$. Since $B^{\prime} \Rightarrow A^{\prime}$, therefore $B m$-implies $A$.

One might try insisting furthermore that differentiations be injective, but we can adapt the counterexample just given by adding only that $c, d, e, f$ be distinct.

I have been unable to solve this problem by internally adjusting the differentiation concept that the manifest consequence definition invokes. The difficulty seems to be that the definition has the form "for every $\cdots$ of $X$, there's a $\cdots$ of $A$ such that $\sim$ ", and the strength of a statement of this form is not straightforwardly related to the strength of the condition $\sim$. However, we might try instead to revise the manifest consequence definition itself. Consider the first suggestion of Footnote $11,{ }^{26}$ requiring that every object that occurs in the differentiated conclusion occur already in at least one of the differentiated hypotheses. As previously observed, cardinality issues show that we cannot consider only the strong differentiations, but need to allow weak ones as well. We thus obtain another account: 
$A$ is a manifest consequence of $X$ iff for every differentiation $\bar{X} \sigma^{\prime}$ of $X$, there's a differentiation $\bar{A} \tau^{\prime}$ of $A$ such that $\operatorname{ran}\left(\tau^{\prime}\right) \subseteq \operatorname{ran}\left(\sigma^{\prime}\right)$.

However, the same kind of counterexample still applies, even under the further requirement that differentiations be conservative.

- Take $B=\ulcorner a=a\urcorner$, and take $A=\left\ulcorner T_{a} \wedge T_{b} \wedge a=b\right\urcorner$. Suppose $B^{\prime}=\ulcorner c=d\urcorner$ is a differentiation of $B$. It suffices to pick $A^{\prime}=\left\ulcorner T_{c} \wedge T_{d} \wedge c=d\right\urcorner$ as the differentiation of $A$.

\subsection{DEVELOPING THE DIFFERENTIATION-BASED APPROACH}

One might pursue further the strategy to appeal to the differentiation $\sigma^{\prime}$ of the hypothesis in generating a constraint on the space of witnessing differentiations $\tau^{\prime}$ of the conclusion. As before, the requirement that differentiations be injective raises cardinality issues, and so we begin by trying to get by without it. Fine's Footnote 11 suggested requiring that the objects that occur in the differentiated conclusion have occurred already in the hypotheses. Although this requirement doesn't quite suffice, we might furthermore insist that whenever some object gives way to another in the differentiated conclusion, that same object must already somewhere have given way to the other in the hypotheses. In other words, we might think of the replacement $\sigma^{\prime} / \sigma$ as 'covering' the space of possible candidates $\tau^{\prime}$. More precisely:

DEFINITION 5. The replacement $\sigma^{\prime} / \sigma$ covers the replacement $\tau^{\prime} / \tau$ provided that whenever $\tau^{\prime}(\alpha) \neq \tau(\alpha)$, then there's a $\beta$ such that $\sigma^{\prime}(\beta)=\tau^{\prime}(\alpha)$ and $\sigma(\beta)=\tau(\alpha)$.

Let us now take differentiation to be the weak notion given by Definition 4 . Then, Fine's first analysis amounts to this: some premises entail a conclusion manifestly, provided that every differentiation of the premises covers at least one of the differentiations of the conclusion which it entails. ${ }^{27}$ It is therefore relative to some choice of base relation $\Rightarrow_{I}$ that this account yields a notion $\Rightarrow_{M I}$ of manifest consequence. Formally,

DEFINITION 6. $\bar{X} \sigma \Rightarrow_{M I} \bar{A} \tau$ iff for every differentiation $\bar{X} \sigma^{\prime}$, there's a differentiation $\bar{A} \tau^{\prime}$ such that $\sigma^{\prime} / \sigma$ covers $\tau^{\prime} / \tau$, where $\bar{X} \sigma^{\prime} \Rightarrow_{I} \bar{A} \tau^{\prime}$.

We now explore the notion just defined. To simplify formulation, we write simply $I, M I$, etc., instead of $\Rightarrow_{I}, \Rightarrow_{M I}$, etc., when these consequence relations are considered as classes of pairs. The first result verifies that indeed, a manifest consequence is a genuine one. In fact, this result is independent of the underlying notion of genuine consequence.

PROPOSITION 1. $M I \subseteq I$. 
Proof. Suppose that $\bar{X} \sigma \Rightarrow_{M I} \bar{A} \tau$. Now in particular, $\bar{X} \sigma$ weakly differentiates itself. Thus, there's a $\tau^{\prime}$ such that $\sigma / \sigma$ covers $\tau^{\prime} / \tau$, and $\bar{X} \sigma \Rightarrow_{I} \bar{A} \tau^{\prime}$. But $\sigma / \sigma$ covers $\tau^{\prime} / \tau$ only if $\tau^{\prime}=\tau$. Hence $\bar{X} \sigma \Rightarrow_{L} \bar{A} \tau$.

Conversely, note that a classical consequence should fail to be manifest only if it involves some recurrence of objects essentially. So in particular, manifest consequence ought to coincide with classical consequence in regard to propositions that contain no objects. Indeed, this is the case, and once again independently of $I$.

PROPOSITION 2. Suppose that no object occurs in $X$. If $X \Rightarrow_{I} A$, then $X \Rightarrow_{M I} A$.

Proof. Suppose that $X \Rightarrow_{I} A$, where $X=\bar{X} \sigma$ and $A=\bar{A} \tau$. Since no object occurs in $X$, therefore if $\bar{X} \sigma^{\prime}$ differentiates $X$, then $\sigma^{\prime}=\sigma$. Hence, it suffices to find a $\tau^{\prime}$ such that $\bar{X} \sigma \Rightarrow_{I} \bar{A} \tau^{\prime}$, where $\sigma / \sigma$ covers $\tau^{\prime} / \tau$. We are forced to take $\tau^{\prime}=\tau$. But $X \Rightarrow_{I} \bar{A} \tau$ by hypothesis. Therefore $X \Rightarrow_{M I} A$.

Nonetheless, the manifest consequence relation over a base relation does not coincide with it, at least when the base relation respects some very weak conditions. For example,

PROPOSITION 3. Suppose $B, B \rightarrow A \Rightarrow_{I} A$ for all $A$ and $B$. Then $I \nsubseteq M I$.

Proof. Let $B$ be $F a$ and let $A$ be $G a$, and let $X$ be the two propositions $B, B \rightarrow A$. Then by hypothesis, $X \Rightarrow_{I} A$. Now, let $X^{\prime}$ be a differentiation $\ulcorner F a, F b \rightarrow G b\urcorner$ of $X$ such that $b \neq a$. Thus $\bar{X} \sigma$ gives way to $\bar{X} \sigma^{\prime}$ where $\sigma=\langle a, a, a\rangle$ and $\sigma^{\prime}=\langle a, b, b\rangle$. Now suppose $\bar{A} \tau^{\prime}$ differentiates $A=\bar{A} \tau$ and that $\sigma^{\prime} / \sigma$ covers $\tau^{\prime} / \tau$. Then either $\tau^{\prime}=\langle a\rangle$ or $\tau^{\prime}=\langle b\rangle$. But in neither case have we $\bar{X} \sigma^{\prime} \Rightarrow_{I} \bar{A} \tau^{\prime}$. Hence $\bar{X} \sigma \nRightarrow_{M I} \bar{A} \tau$.

We have defined manifest consequence relative to a base relation $I$. What happens if we adjust this parameter? A natural feature to expect is that $M I$ be increasing in $I$. That is to say:

PROPOSITION 4. Suppose $I \subseteq J$. Then $M I \subseteq M J$.

Proof. Assume $\bar{X} \sigma \Rightarrow_{M I} \bar{A} \tau$. Let $\bar{X} \sigma^{\prime}$ differentiate $\bar{X} \sigma$. It suffices to find a $\tau^{\prime}$ such that $\sigma^{\prime} / \sigma$ covers $\tau^{\prime} / \tau$ and $\bar{X} \sigma^{\prime} \Rightarrow_{J} \bar{A} \tau^{\prime}$. Well, by the definition of $\Rightarrow_{M I}$ we have a covered $\tau^{\prime}$ such that $\bar{X} \sigma^{\prime} \Rightarrow_{I} \bar{A} \tau^{\prime}$, so that $\bar{X} \sigma^{\prime} \Rightarrow_{J} \bar{A} \tau^{\prime}$ as well. Hence $\bar{X} \sigma \Rightarrow_{M J} \bar{A} \tau$.

PROPOSITION 5. $M(K \cap L) \subseteq M K \cap M L$.

Proof. Immediate from Proposition 4.

This last result raises the question of the converse, which is not obvious. Suppose that $X \Rightarrow_{(M K) \cap(M L)} A$. Then for a given differentiation $X^{\prime}$ of $X$ there 
are covered $A_{1}, A_{2}$ such that $X^{\prime} \Rightarrow_{K} A_{1}$ and $X^{\prime} \Rightarrow_{L} A_{2}$, but there is no reason to suppose we can always achieve $A_{1}=A_{2}$. Indeed, there are counterexamples.

PROPOSITION 6. $M K \cap M L \nsubseteq M(K \cap L)$.

Proof. It suffices to find a sequence $X$ and a formula $A$ such that every differentiation of $X$ covers a differentiation of $A$ that it $K$-entails, and every differentiation of $X$ covers a differentiation of $A$ that it $L$-entails, but not every differentiation of $X$ covers a differentiation of $A$ that it both $K$-entails and $L$-entails. Let $X$ consist of the single proposition

$$
a \neq a \wedge \exists x(x=a \wedge \forall y(F y \leftrightarrow y=x) \wedge \forall y(G y \leftrightarrow y \neq x)),
$$

and let $A$ be the proposition

$$
\forall x(x=a \rightarrow(G x \vee(F x \leftrightarrow(\forall y H y \rightarrow H b))))
$$

for some constant $b \neq a$.

Consider a differentiation $\bar{X} \sigma^{\prime}$ of $X$. On the one hand, if $\sigma^{\prime}(0)=\sigma^{\prime}(1)$, then by setting $\tau^{\prime}=\tau$ we ensure $\bar{X} \sigma^{\prime} \Rightarrow_{K \cap L} \bar{A} \tau^{\prime}$ with $\tau^{\prime}$ covered. On the other hand, suppose $\sigma^{\prime}(0) \neq \sigma^{\prime}(1)$. And take $\bar{X} \sigma^{\prime}$ to cover the differentiation $\bar{A} \tau^{\prime}$. Note that this implies $\tau^{\prime}(1)=\tau(1)$ since $b=\tau(1)$ does not occur in $X$. If $\tau^{\prime}(0) \neq \sigma^{\prime}(2)$, then $\bar{X} \sigma^{\prime} \Rightarrow_{K} \bar{A} \tau^{\prime}$, but also $\bar{X} \sigma^{\prime} \nRightarrow_{L} \bar{A} \tau^{\prime}$. If $\tau^{\prime}(0)=\sigma^{\prime}(2)$, then $\bar{X} \sigma^{\prime} \Rightarrow_{L} \bar{A} \tau^{\prime}$; furthermore, in the subcase where $\sigma^{\prime}$ has been chosen so that $\sigma^{\prime}(2) \neq \tau(1)$ we must also have $\tau^{\prime}(1) \neq \sigma^{\prime}(2)$, which yields $\bar{X} \sigma^{\prime} \nRightarrow_{K} \bar{A} \tau^{\prime}$.

Writing $I \longrightarrow J$ to mean $I \subsetneq J$ we obtain a complete comparison:

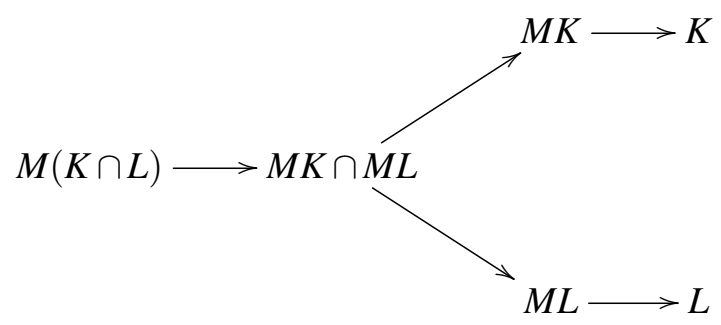

\subsubsection{Trouble with $\Rightarrow_{M K}$}

The notion of manifest consequence is intended to capture the consequences a reasoner could ideally draw under some deficient grasp on the identity of objects. Now, we remarked earlier that $\Rightarrow_{K}$ differs from $\Rightarrow_{L}$ in that $\Rightarrow_{K}$ validates truths of distinctness. In fact the feature propagates through to $\Rightarrow_{M K}$.

PROPOSITION 7. If $a \neq b$, then $\emptyset \Rightarrow_{M K}\ulcorner a \neq b\urcorner$.

Proof. Immediate from $\emptyset \Rightarrow_{K}\ulcorner a \neq b\urcorner$ by Proposition 2 .

While informativeness of identities has been an abiding focus of philosophy of language, facts of distinctness can be no less surprising. For example, 
if distinctness were epistemically transparent, then it would be impossible to steal someone's identity. Unfortunately, identity theft is common. ${ }^{28} \mathrm{We}$ should therefore abandon $\Rightarrow_{M K}$ as an analysis of manifest consequence, and shift our attention to $\Rightarrow_{M L}$ and its subrelations.

\subsubsection{A postulate}

The analysis of manifest consequence as $\Rightarrow_{M L}$ still suffers from a technical flaw. The point of generalizing over differentiations of the hypotheses is to ensure that conclusions be drawn compatibly with the circumstance that the hypotheses even contain no recurrences whatsoever. For example, let $\left\langle a_{\alpha}\right.$ : $\alpha\langle\gamma\rangle$ be a sequence of not necessarily distinct elements of $\mathcal{l}$, and let $X$ be a sequence of propositions that contains each pair $\left\ulcorner a_{\alpha} \neq a_{\beta}\right\urcorner$ such that $\alpha<\beta<\gamma$. In this case, it may well be that 'for all we know', $a_{\alpha} \neq a_{\beta}$ for all such $\alpha, \beta$; hence for all we know, the sequence $X$ is satisfiable. This suggests that $X$ ought not to be manifestly inconsistent. But, if we apply the definition of $\Rightarrow_{M L}$, then there needs to exist a differentiation $X^{\prime}$ of $X$ such that $X^{\prime} \nRightarrow_{L} \perp$. Of course, $\ulcorner a \neq a\urcorner \Rightarrow_{L} \perp$ for any $a$, and consequence relations that lack this property seem to be precisely among those we are now trying to analyze. Therefore, we need to be sure that there exists a differentiation $X^{\prime}$ of $X$ that is injective. No such differentiation exists if $\operatorname{Card}(\gamma)>\operatorname{Card}(\mathcal{U})$.

This problem seems to be merely technical, because it arises only in case a list of hypotheses is equinumerous with the totality of objects in the universe. So, I suggest a solution by fiat:

POSTULATE 1. If $\bar{X} \sigma$ is a list of hypotheses, then $\operatorname{Card}(|\sigma|)+k<\operatorname{Card}(\mathcal{U})$, for all $k<\omega .^{29}$

\subsubsection{Another characterization of $\Rightarrow_{M L}$}

Upon shifting to $\Rightarrow_{L}$ as the base relation, the notion of $M$-consequence can now be seen from another perspective. The differentiation-based account of manifest consequence flows from a conceit that the objects in some list of hypotheses might be swapped around, perhaps by an evil demon. But one might also understand a consequence to be manifest in case every occurrence of an object in the hypotheses behaves like a distinct "take" on that object. Once the original objects have been replaced by distinct takes, we then simply reason

classically, treating the "takes" like ordinary constants, and at the end of the process, undo the original replacement. It can now be shown that these two informal characterizations coincide. In one direction the result is immediate:

PROPOSITION 8. Suppose that $\bar{X} \sigma \Rightarrow_{M L} \bar{A} \tau$. Then there is an injective differentiation $\mathrm{\imath}$ of $\sigma$, and a differentiation $\xi$ of $\tau$, such that $\mathrm{\imath} / \sigma$ covers $\xi / \tau$, and $\bar{X} \mathbf{\imath} \Rightarrow_{L} \xi$.

Proof. The existence of 1 follows from Postulate 1, and the existence of $\tau$ then follows by the hypothesis that $\bar{X} \sigma \Rightarrow_{M L} \bar{A} \tau$. 
The converse requires some argument.

PROPOSITION 9. Suppose that $\bar{X} \mathrm{\imath} \Rightarrow_{L} \bar{A} \xi$, where $\mathrm{\imath}$ is injective and $1 / \sigma$ covers $\xi / \tau$. Then $\bar{X} \sigma \Rightarrow_{M L} \bar{A} \tau$.

Proof. Let $\sigma^{\prime}$ be a differentiation of $\sigma$. It suffices to find a differentiation $\tau^{\prime}$ of $\tau$ such that $\sigma^{\prime} / \sigma$ covers $\tau^{\prime} / \tau$, with $\bar{X} \sigma^{\prime} \Rightarrow_{L} \bar{A} \tau^{\prime}$. By Postulate 1, we may assume that $\operatorname{Ran}(\boldsymbol{l} \frown \xi) \cap \operatorname{Ran}(\sigma \frown \tau)=\emptyset$. Since $l$ is injective, there's a function $f$ such that $f \circ \mathbf{l}=\sigma^{\prime}$. Take $f^{\prime}$ such that $f^{\prime}(a)=f(a)$ if there's a $\beta<|\sigma|$ such that $\mathrm{l}(\beta)=a$, and $f^{\prime}(a)=a$ otherwise. Let $\tau^{\prime}$ be the sequence such that $\sigma^{\prime} \frown \tau^{\prime}=f^{\prime} \circ(1 \frown \xi)$. Since $\sigma^{\prime} \frown \tau^{\prime}$ is a substitution instance of $\imath \frown \xi$ and since $\Rightarrow_{L}$ respects substitution, it therefore suffices to show that $\sigma^{\prime} / \sigma$ covers $\tau^{\prime} / \tau$.

So suppose that $\tau^{\prime}(\alpha) \neq \tau(\alpha)$ for some $\alpha<|\tau|$. Now, further assume on the one hand that $\left(f^{\prime} \circ \xi\right)(\alpha)=\xi(\alpha)$. Since $\mathrm{\imath} / \sigma$ covers $\xi / \tau$, it follows that there's a $\beta<|\sigma|$ such that $\sigma(\beta)=\tau(\alpha)$ and $\mathrm{l}(\beta)=\xi(\alpha)$; the latter gives $\sigma^{\prime}(\beta)=(f \circ \mathfrak{l})(\beta)=\left(f^{\prime} \circ \mathfrak{l}\right)(\beta)=\left(f^{\prime} \circ \xi\right)(\alpha)=\tau^{\prime}(\alpha)$.

Assume on the other hand that $\left(f^{\prime} \circ \xi\right)(\alpha) \neq \xi(\alpha)$ so by choice of $f^{\prime}$, there's a $\beta<|\sigma|$ such that $\mathrm{t}(\beta)=\xi(\alpha)$. Then $\tau^{\prime}(\alpha)=\left(f^{\prime} \circ \xi\right)(\alpha)=(f \circ$ $\xi)(\alpha)=(f \circ \mathrm{l})(\beta)=\sigma^{\prime}(\beta)$. Moreover, by the choice of $\mathrm{\imath}$ and $\xi$, it follows that $\xi(\alpha) \neq \tau(\alpha)$, so since $\mathrm{l} / \sigma$ covers $\xi / \tau$ there is a $\gamma<|\sigma|$ such that $\xi(\alpha)=\mathfrak{l}(\gamma)$ and $\tau(\alpha)=\sigma(\gamma)$. Thus $\mathfrak{\imath}(\beta)=\xi(\alpha)=\mathfrak{l}(\gamma)$, and the injectivity of $\mathrm{l}$ now gives $\beta=\gamma$, so that $\sigma(\beta)=\tau(\alpha)$, as desired.

\section{The coordination-based analysis}

In the previous section we developed an account of those consequences which can be drawn regardless of the identities of the objects that occur in some given hypotheses. ${ }^{30}$ Because, on this account, the negation of a true identity does not manifestly lead to contradiction, it looks like we have accommodated the point that identities need not be trivial. Of course, Frege's puzzle is not that no identity is trivial, but rather that some are and some are not. Indeed, although on the one hand we have

$$
\ulcorner a \neq a\urcorner \nRightarrow_{M} \perp,
$$

still, by Proposition 2, we also have

$$
\emptyset \Rightarrow_{M}\ulcorner a=a\urcorner .
$$

But now the analysis looks rather ambivalent: one proposition is not trivial, in that its negation does not $M$-entail absurdity, and yet that same proposition is trivial, because it is an $M$-consequence of the empty set. In other words, we have got some more explaining to do. 
Actually we have now reached the situation in which Fine exhorts us to find ourselves at the beginning of his book: to feel a need to attend not just to informativeness but also to triviality, thereby raising the question what makes it possible for two utterances to say the same thing. This is what semantic relationism with its central notion of strict coreference is meant to address. Now, one might propose that strict coreference is not a genuinely semantic phenomenon, but rather something to be explained by, for example, refining the identity conditions of proper names, or attending carefully to the nature of their occurrences. Of course, semantic properties of expressions must depend to some extent on syntactic properties, since expressions are syntactic items in the first place. But, Fine argues that syntactic properties cannot be entirely constitutive of strict coreference. For example, typographical identity would be neither necessary nor sufficient. Rather, Fine concludes: "there is some underlying phenomenon, not resting upon the expressions being the same, in virtue of which they represent the object as the same."31

Thus, Fine instead proposes a properly semantic explanation: that names corefer strictly when their meanings are 'coordinated'. More precisely, on this view, a sentence would express not just a Russellian proposition, but a coordinated proposition, which consists of a Russellian proposition together with an equivalence relation on its occurrences of objects. ${ }^{32}$ Thus, the relata of coordination are not expressions but rather the occurrence of the meanings of those expressions within the total meaning of the body of discourse. ${ }^{33} \mathrm{In}$ this way, Fine can allow that "Cicero is Cicero" and "Cicero is Tully" may differ in meaning even though the constituent names, considered intrinsically, all share the same meaning: the difference in meaning corresponds rather to the difference that only the first sentence expresses a proposition in which the two occurrences of a man are coordinated. Actually, Fine allows a coordination scheme to extend over an entire body of propositions. In this way, the sentences "Cicero is Roman" and "Tully is Roman" might agree with respect to their intrinsic semantic properties, yet differ with respect to their coordinating links to propositions expressed by sentences elsewhere in the discourse. $^{34}$

Coordination is intended to serve not just an account of the semantics of sentences but a more general account of cognitive states. So, not only would coordinated sentences be expressed by propositions, they would also be objects of belief, knowledge, and intention. ${ }^{35}$ Indeed, this ambition to generality may also motivate the dismissal of syntactical explanations of strict coreference. ${ }^{36}$ So there is quite a wide variety of sources for the coordination relationship: coordination would mark not only strict synonymy of expressions in sentences, but also all kinds of cross-reference that pervade reasoning, perception and belief. It is therefore natural to expect that anaphoric bonds may develop between representations of different objects. ${ }^{37}$ So, although the strict coreference of two names implies that their meanings are coordinated, 
conversely the variety of natural roles for coordination suggests that through some confusion the meanings of two names may be coordinated although the names do not corefer. ${ }^{38}$ Say that a coordination of occurrences of objects over a sequence of propositions is sound if it relates no occurrences of distinct objects. In the following discussion, we develop an analysis of coordination over occurrences of objects in propositions that is independent of the assumption of soundness, and therefore identify precisely some effects of this assumption.

Throughout much of the discussion in $S R$, Fine maintains the assumption that coordination be an equivalence relation. However, it is unclear that this property is secured by the way that language unfolds. As Fine points out, it may be a semantic fact about one expression that it means the same as some preceding expression, while the preceding expression means what it does regardless of what anybody says later. Such asymmetries may induce cognitive phenomena which a semantic theory of coordination ought also to explain. ${ }^{39}$ Perhaps only of ideal representational circumstances should we assume that no essential generality is lost by assuming that the coordination relation is an equivalence. However, this assumption simplifies the conceptual situation considerably. So we maintain it in what follows.

\subsection{MODELLING COORDINATION}

The relationship of coordination over the occurrences of objects in propositions can be modelled following the approach developed above for keeping track of multiplicity of occurrences. Accordingly, a sequence $X$ of propositions over the source $\mathcal{U}$ of constituents is once again represented canonically as $\bar{X} \sigma$, where $\bar{X}$ is the nice sequence associated with $X$, and $\sigma$ is a sequence of elements of $\mathcal{U}$. Since there is exactly one isomorphism between the order type $|\sigma|$ of $\sigma$ and the naturally well-ordered occurrences of individuals in $X$, we can use $|\sigma|$ to define a coordination scheme over $X$ : a coordination scheme would be a relation $C$ on $|\sigma|$.

As noted, we assume that a coordination is an equivalence. Elaborating the suggestion of Fine's endnote, the relation $C$ determines a sequence $\mu$ whose $\alpha$ th term is the least element of the $\mathcal{C}$-equivalence class of $\alpha$. Conversely, an ordinal-valued sequence $\mu$ determines an equivalence relation $\mathcal{C}$ as the relation that holds between $\alpha, \beta<|\sigma|$ if and only if $\mu(\alpha)=\mu(\beta)$. This leads to a definition:

DEFINITION 7. If $X=\bar{X} \sigma$ is a sequence of propositions, then a coordination for $X$ is a sequence $\mu:|\sigma| \rightarrow|\sigma|$ such that for all $\alpha<|\sigma|$, there's a $\beta \leq \alpha$ such that $\mu(\alpha)=\mu(\beta)=\beta$.

Of course, any mapping $|\sigma| \rightarrow|\sigma|$ determines an equivalence relation on its domain. The extra condition on $\mu$ gives the following useful result. 
PROPOSITION 10. Let $\mu$ be a coordination scheme. Then $\mu \circ \mu=\mu$.

Proof. Take $\alpha<|\mu|$; then the $\beta$ of Definition 7 gives $\mu(\mu(\alpha))=\mu(\mu(\beta))=$ $\mu(\beta)=\mu(\alpha)$.

As mentioned earlier, it may arise through confusion that distinct objects may be represented "as the same object" throughout various occurrences in some sequence of propositions. In the formalism just given, if $\mu$ is a coordination for $\sigma$, such confusions will be represented by the circumstance that for some $\alpha, \beta<|\sigma|$ we have $\mu(\alpha)=\mu(\beta)$ although $\sigma(\alpha) \neq \sigma(\beta)$. The scheme $\mu$ will be called $\sigma$-sound when no such confusions arise.

Regarding the notion of soundness we have the following.

PROPOSITION 11. Suppose that $\mu$ is $\sigma$-sound. Then $\sigma \circ \mu=\sigma$.

Proof. Take $\alpha<|\mu|$. By Proposition 10, $\mu(\mu(\alpha))=\mu(\alpha)$. The $\sigma$-soundness of $\mu$ now gives $\sigma(\mu(\alpha))=\sigma(\alpha)$.

\subsection{FAITHFULNESS}

In his endnote 14 of Chapter 2, Fine returns briefly to the notion of manifest consequence, remarking that this concept can be explained in terms of a more general concept, that of consequence relative to a coordination scheme-or, as Fine puts it, of coordinated inference.

Thus the inference from $X$ to $A$ will be manifestly valid if for every coordination scheme $C$ on $X$, there's an extension of it to $X, A$ under which the argument from $X$ to $A$ is valid. [Quoted with relettering from 136n14.]

To spell out this idea, we first need to explicate the idea of an extension of an equivalence over $|\sigma|$ to an equivalence over $|\sigma \frown \tau|$. Clearly if $C^{\prime}$ is such an extension of an equivalence $\mathcal{C}$, then $C^{\prime}$ should be an equivalence relation as well, and moreover we ought to have $C \subseteq C^{\prime}$. We also need to insist that $C^{\prime}$ is conservative, in the sense that the restriction of $C^{\prime}$ to $|\sigma|$ be equal to $\mathcal{C}$, since otherwise we could always pick for $\mathcal{C}^{\prime}$ the maximal or maximal-sound coordination, yielding in either case far too weak a result. Supposing we continue to represent $C$ as an ordinal-valued sequence $\mu$, then conservativeness is ensured automatically by representing the extension $C^{\prime}$ as a concatenation $\mu \frown v$. However, at this point we may again be stuck with the manifest validity of $\ulcorner a=b\urcorner$, because nothing so far prevents $C^{\prime}$ from conflating objects that do not yet occur in $\mathcal{C}$. One might insist that $C^{\prime}$ be sound, but this would be disappointing, because it would prevent us from characterizing the consequences that can be drawn relative to unsound schemes on $|\sigma|$. Rather, the desired idea is that all conflations committed by the extension $C^{\prime}$ can be traced back to conflations committed by $C$. More precisely, if $C^{\prime}$ conflates occurrences of $c$ and $d$, then $C^{\prime}$ also coordinates 
those occurrences with occurrences that $\mathcal{C}$ conflated already. Whenever the conflations of $C^{\prime}$ are always "excused" in this way, $C^{\prime}$ will be said to be faithful. More precisely:

DEFINITION 8. Let $\mu$ be a coordination for $\bar{X} \sigma$; then $a$ faithful extension of $\mu$ to $\bar{X} \sigma \frown \bar{A} \tau$ is a coordination $\mu \frown v$ such that for all $\gamma, \delta<|\sigma \frown \tau|$, if $(\mu \frown v)(\gamma)=(\mu \frown v)(\delta)$ while $(\sigma \frown \tau)(\gamma) \neq(\sigma \frown \tau)(\delta)$, then there are "excusers" $\alpha, \beta<|\sigma|$ such that

$$
\begin{aligned}
& -(\mu \frown v)(\gamma)=(\mu \frown v)(\alpha),(\mu \frown v)(\delta)=(\mu \frown v)(\beta), \text { and } \\
& -(\sigma \frown \tau)(\gamma)=(\sigma \frown \tau)(\alpha),(\sigma \frown \tau)(\delta)=(\sigma \frown \tau)(\beta) .
\end{aligned}
$$

In other words, using _ and - -- to mean agreement with respect to $\sigma \frown \tau$ and $\mu \frown \nu$ respectively, then the condition on $\gamma, \delta$ comes to

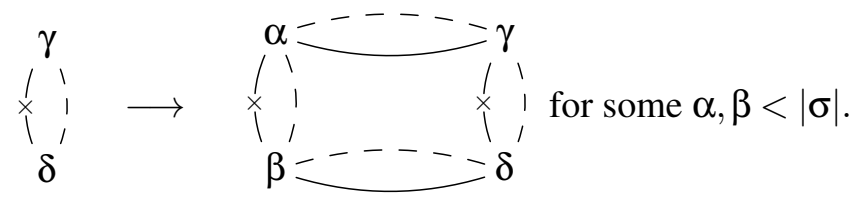

Regarding the notion of faithfulness we have now ${ }^{40}$

PROPOSITION 12. A faithful extension of a sound scheme is sound.

Proof. Suppose $\mu$ is sound, and suppose $\mu \frown v$ extends $\mu$ faithfully to $(\bar{X} \frown$ $\bar{A})(\sigma \frown \tau)$. Now let $\gamma, \delta$ be such that $(\mu \frown v)(\gamma)=(\mu \frown v)(\delta)$ but $(\sigma \frown$ $\tau)(\gamma) \neq(\sigma \frown \tau)(\delta)$. By faithfulness, we have excusers $\alpha, \beta<|\sigma|$ such that $(\mu \frown v)(\alpha)=(\mu \frown v)(\beta)$ although $(\sigma \frown \tau)(\alpha) \neq(\sigma \frown \tau)(\beta)$, contradicting the soundness of $(\mu \frown v)$.

\subsection{Coordinated CONSEQUence}

According to Fine, a speaker who grasps the meaning of an English sentence thereby recognizes not just the Russellian proposition expressed but also a coordination scheme over that proposition. The coordination scheme serves in part to determine the cognitive value of what is expressed by the sentence. So in particular, the coordination scheme should help to determine whether or not the sentence says something whose negation transparently implies a contradiction. So Fine's account of cognitive significance requires a notion of logical consequence that is relativized to schemes of coordination. Indeed, the previously quoted analysis in Footnote 14 takes the manifest consequences of some hypotheses to be those propositions which can be inferred under every possible scheme. The endnote continues as follows: 
[V]alidity for a coordinated inference is explained in terms of the validity of an inference in which each occurrence of an individual is replaced by its equivalence class under the coordination scheme.... [136n14]

Thus, on Fine's account, the logical consequences of some hypotheses $X$, relative to a scheme $\mathcal{C}$, are, roughly speaking, the consequences which can be reached after the objects in $X$ have given way to proxies; different occurrences of objects give way to different proxies precisely when the occurrences are uncoordinated. It seems clear that Fine intends the same underlying consequence relation as was intended previously, so for simplicity we take it to be $L$-consequence. ${ }^{41}$

For technical reasons, we model this notion of a proxy using not the equivalence classes of ordinals which correspond to occurrences, but rather the least members of those classes. Let $\mathcal{L}_{|\mu \frown v|}$ be the language that results from $\mathcal{L}_{\emptyset}$ by adding to its nonlogical vocabulary as terms the ordinals up to $|\mu \frown v|$. We consider as structures for $\mathcal{L}_{|\mu \frown v|}$ simply the $L$-structures already defined, and the previously defined notion of $L$-consequence carries over.

The resulting analysis of coordinated consequence is now as follows:

DEFINITION 9. Let $\mu$ be a coordination for the sequence $\bar{X} \sigma$ of propositions. Then $\bar{X} \sigma$ c-entails $\bar{A} \tau$ under $\mu$ provided that there's a faithful extension $\mu \frown v$ of $\mu$ such that $\bar{X} \mu \Rightarrow_{L} \bar{A} v$.

As Fine's sketch indicated, the notion of coordinated consequence gives rise immediately to a notion of manifest consequence:

DEFINITION 10. $X \Rightarrow_{N} A$ iff $X$ c-entails $A$ under every coordination $\mu$ of $X$.

While the definition of $N$-consequence involves generalizing over the various relativizations of $c$-consequence, in fact the generality is inessential. To find the $N$-consequences of $\bar{X} \sigma$, it suffices to find its $c$-consequences under a coordination that is "minimal." Write $l_{\alpha}$ for the identity function on the ordinal $\alpha$. (We will drop the subscript when the appropriate order type is obvious.)

PROPOSITION 13. $\bar{X} \sigma \Rightarrow_{N} A$ iff $\bar{X} \sigma$ c-entails $B$ under $\mathbf{l}_{|\sigma|}$.

Proof. The left-to-right direction follows trivially from the definition of $N$-consequence. So assume that $\bar{X} \sigma c$-entails $A$ under $\boldsymbol{l}_{|\sigma|}$. And let $\mu$ be a coordination for $X$. By hypothesis, there's a faithful extension $\mathbf{l}_{|\sigma|} \frown \xi$ of $\mathbf{l}_{|\sigma|}$ such that $\bar{X} 1_{|\sigma|} \Rightarrow_{L} \bar{A} \xi$. Now, let $v$ be the unique sequence such that $\mu \frown v=$ $\left(\mu \frown \mathfrak{l}_{|\tau|}\right) \circ\left(\mathfrak{l}_{|\sigma|} \frown \xi\right)$. Thus $(\bar{X} \frown \bar{A})(\mu \frown v)$ has the form $(\bar{X} \frown \bar{A})\left(f \circ\left(\mathbf{l}_{|\sigma|} \frown\right.\right.$ $\xi)$ ) for some $f$. Since $\Rightarrow_{L}$ respects substitutivity, it follows that $\bar{X} \mu \Rightarrow_{L} \bar{A} v$. It remains to verify that $\mu \frown v$ is faithful. We will write $\xi^{\prime}=\left(\mathfrak{l}_{|\sigma|} \frown \xi\right)$.

Consider an arbitrary $\eta \geq|\sigma|$. Observe that since $\xi^{\prime}$ is $\sigma$-sound, it follows by Proposition 11 that

$$
(\sigma \frown \tau)\left(\xi^{\prime}(\eta)\right)=(\sigma \frown \tau)(\eta) .
$$


Similarly, by Proposition 10 we have

$$
(\mu \frown v)\left(\xi^{\prime}(\eta)\right)=\left(\mu \frown \mathbf{l}_{|\tau|}\right)\left(\xi^{\prime}\left(\xi^{\prime}(\eta)\right)\right)=\left(\mu \frown \mathbf{l}_{|\tau|}\right)\left(\xi^{\prime}(\eta)\right)=(\mu \frown v)(\eta) .
$$

Now, let $\gamma, \delta$ be such that $(\mu \frown v)(\gamma)=(\mu \frown v)(\delta)$, but $(\sigma \frown \tau)(\gamma) \neq(\sigma \frown$ $\tau)(\delta)$. Following the definition of faithfulness, we seek excusers $\alpha$ and $\beta$.

Suppose $\gamma, \delta<|\sigma|$. By the observations above, it suffices to put $\alpha=\gamma$ and $\beta=\delta$.

Suppose $\gamma<|\sigma| \leq \delta$. If $\xi^{\prime}(\delta)<|\sigma|$, then it suffices to put $\alpha=\xi^{\prime}(\gamma)$. If $|\sigma| \leq \xi^{\prime}(\delta)$, then $(\mu \frown v)(\gamma)<|\sigma| \leq \xi^{\prime}(\delta)<(\mu \frown v)(\delta)$, contradicting $(\mu \frown$ $v)(\gamma)=(\mu \frown v)(\delta)$.

Finally, suppose that $|\sigma| \leq \gamma, \delta$. If $\xi^{\prime}(\gamma), \xi^{\prime}(\delta)<|\sigma|$, then it suffices to put $\alpha=\xi^{\prime}(\gamma)$ and $\beta=\xi^{\prime}(\delta)$. If $\xi^{\prime}(\gamma)<|\sigma| \leq \xi^{\prime}(\delta)$, then $(\mu \frown \nu)(\gamma)<|\sigma| \leq(\mu \frown$ $v)(\delta)$, a contradiction. If $|\sigma| \leq \xi^{\prime}(\gamma), \xi^{\prime}(\delta)$, then $\xi^{\prime}(\gamma)=(\mu \frown v)(\gamma)=(\mu \frown$ $v)(\delta)=\xi^{\prime}(\delta)$; but since $(\sigma \frown \tau)(\gamma) \neq(\sigma \frown \tau)(\delta)$, the soundness of $\xi^{\prime}$ implies $\xi^{\prime}(\gamma) \neq \xi^{\prime}(\delta)$, which is again a contradiction.

In setting up the notion of a coordination scheme we allowed that a scheme be "unsound" in the sense that it may conflate distinct objects. The next result delimits the effects of this liberality.

PROPOSITION 14. A sequence $X$ c-entails $A$ under every coordination iff $X$ c-entails A under every sound coordination.

Proof. The left-to-right is trivial. So assume the right-hand side. Clearly, $\mathbf{l}_{|\sigma|}$ is sound. So by hypothesis, $X c$-entails $A$ under $\mathbf{1}_{|\sigma|}$, and the conclusion follows by Proposition 13.

However, if we are confused about our hypotheses then reasoning may make matters worse.

PROPOSITION 15. There are propositions $B$ and $A$ and a coordination scheme $\mu$ such that $B$ c-entails $A$ under $\mu$ but $B \nRightarrow_{L} A$.

Proof. Let $B$ be a proposition $\bar{C}\langle a, b\rangle$ such that $a \neq b$, and let $A$ be the proposition $\ulcorner a=b\urcorner$. Clearly, $B \neq_{L} A$. But let $\mu$ be the coordination $\langle 0,0\rangle$. Then $\mu \frown v=\langle 0,0,0,0\rangle$ is a faithful extension of $\mu$ to $B \frown A$, and $\bar{B} \mu \Rightarrow_{L} \bar{A} v$. Hence, $B c$-entails $A$ under $\mu$.

Nonetheless, if we are unconfused, then we are safe. ${ }^{42}$

PROPOSITION 16. Let $\mu$ be sound. If $X$ c-entails A under $\mu$, then $X \Rightarrow_{L} A$.

Proof. Suppose that $\mathcal{M}=_{L} \bar{X} \sigma$ but that $\mathcal{M} \not \forall_{L} \bar{A} \tau$. Now let $v$ be a faithful extension of $\mu$. By Proposition 12, $\mu \frown v$ is sound. So by Proposition 11 , there's a function $h$ such that $h \circ(\mu \frown v)=\sigma \frown \tau$. Cut down $\mathcal{M}$ to $\mathcal{L}_{0}$; and now expand the result to an $\mathcal{M}^{\prime}$ for $\mathcal{L}_{|\mu \frown v|}$, in such a way that 
$((\mu \frown v)(\alpha))^{\mathcal{M}^{\prime}}=h \circ(\mu \frown v)$ for all $\alpha<|\sigma|$. Then $\mathcal{M}^{\prime} \models_{L} \bar{X} \mu$ but $\mathcal{M}^{\prime} \mid \models_{L} A v$. But $\nu$ was an arbitrary faithful extension of $\mu$; hence $X$ does not $c$-entail $A$ under $\mu$.

As with $M$-consequence, we have the fulfillment of a minimal adequacy condition:

PROPOSITION 17. $N L \subseteq L$.

Proof. Suppose that $X \Rightarrow_{N L} A$. Then $X c$-entails $A$ under $\mathbf{v}_{|\sigma|}$. But $\mathbf{l}_{|\sigma|}$ is sound. So by Proposition 13, $X \Rightarrow_{L} A$.

We conclude this section by comparing the coordination-based analysis of manifest consequence with the differentiation-based analysis given in $\S 3$.

PROPOSITION 18. $N L \subseteq M L$.

Proof. Suppose that $X \Rightarrow_{N L} A$, where $X=\bar{X} \sigma$ and $A=\bar{A} \tau$. Let $\sigma^{\prime}$ be an arbitrary differentiation of $\sigma$. It suffices to find a differentiation $\tau^{\prime}$ of $\tau$ such that $\sigma^{\prime} / \sigma$ covers $\tau^{\prime} / \tau$, where $\bar{X} \sigma^{\prime} \Rightarrow_{L} \bar{A} \tau^{\prime}$.

Since $X \Rightarrow_{N} A$, there is a faithful $\xi$ such that $\bar{X} 1 \Rightarrow_{L} \bar{A} \xi$. Write $\xi^{\prime}=1 \frown \xi$. Since $\xi^{\prime}$ is sound, it follows by Proposition 11 that there is a $g$ such that $\left(g \circ \xi^{\prime}\right)(\alpha)=\left\langle\xi^{\prime}(\alpha),(\sigma \frown \tau)(\alpha)\right\rangle$ for all $\alpha<|\sigma \frown \tau|$. Write $\phi=g \circ \xi^{\prime}$. We now aim to construct a function $f$ such that the desired $\tau^{\prime}$ will satisfy $\sigma^{\prime} \frown$ $\tau^{\prime}=f \circ \phi$. First, suppose that there is a $\beta<|\sigma|$ such that $\phi(\alpha)=\phi(\beta)$. Then $\phi(\alpha)=\langle\beta, \sigma(\beta)\rangle$, and so we may set $f(\phi(\alpha))=\sigma^{\prime}(\beta)$. On the other hand, suppose that there is no such $\beta$. Since $\phi(\alpha)$ has the form $\left\langle\xi^{\prime}(\alpha),(\sigma \frown \tau)(\alpha)\right\rangle$, we may here take $f(\phi(\alpha))=(\sigma \frown \tau)(\alpha)$.

It is clear that $\operatorname{dom}(f \circ \phi)=|\sigma \frown \tau|$, and that $(f \circ \phi) \uparrow|\sigma|=\sigma^{\prime}$. Hence there exists a $\tau^{\prime}$ such that $\sigma^{\prime} \frown \tau^{\prime}=f \circ \phi$. Moreover, if $\left(\sigma^{\prime} \frown \tau^{\prime}\right)(\alpha) \neq(\sigma \frown \tau)(\alpha)$, then there is a $\beta<|\sigma|$ such that $(\sigma \frown \tau)(\alpha)=\sigma(\beta)$ and $\left(\sigma^{\prime} \frown \tau^{\prime}\right)(\alpha)=\sigma^{\prime}(\beta)$. Hence $\sigma^{\prime} / \sigma$ covers $\tau^{\prime} / \tau$, as desired.

Suppose now that $\mathcal{M} \models_{L} \bar{X} \sigma^{\prime}$ but $\mathcal{M} \not \models_{L} \bar{A} \tau^{\prime}$. Cut down $\mathcal{M}$ to $\mathcal{L}_{\emptyset}$ and then expand the result to obtain an $\mathcal{M}^{\prime}$ such that $\left(\xi^{\prime}(\alpha)\right)^{\mathcal{M}^{\prime}}=f\left(g\left(\xi^{\prime}(\alpha)\right)\right)=$ $\left(\sigma^{\prime} \frown \tau^{\prime}\right)(\alpha)$ for all $\alpha<|\sigma \frown \tau|$. Then $\mathcal{M}^{\prime} \models_{L} \bar{X}$ i but $\mathcal{M}^{\prime} \mid \models_{L} \bar{X} \xi$, which is a contradiction.

It turns out that the converse of Proposition 18 holds too.

PROPOSITION 19. $M L \subseteq N L$.

Proof. Let $\bar{X} \sigma \Rightarrow_{M L} \bar{A} \tau$. By Postulate 1, there is an injective differentiation $\sigma^{\prime}$ of $\sigma$ such that if $\sigma^{\prime}(\alpha) \neq \sigma(\alpha)$, then $\sigma^{\prime}(\alpha) \neq(\sigma \frown \tau)(\beta)$ for all $\alpha, \beta$ such that $\alpha<|\sigma| \leq \beta<|\sigma \frown \tau|$. By hypothesis there exists a $\tau^{\prime}$ such that $\sigma^{\prime} / \sigma$ covers $\tau^{\prime} / \tau$ with $\bar{X} \sigma^{\prime} \Rightarrow_{L} \bar{A} \tau^{\prime}$. We first argue that $\sigma^{\prime} \frown \tau^{\prime}$ is a faithful extension of $\sigma^{\prime}$. 
Suppose $\tau^{\prime}(\gamma)=\tau^{\prime}(\delta)$ although $\tau(\gamma) \neq \tau(\delta)$. Without loss of generality, assume $\tau^{\prime}(\gamma) \neq \tau(\gamma)$. Since $\tau^{\prime}$ is covered, we must have $\alpha<|\sigma|$ such that $\tau^{\prime}(\gamma)=\sigma^{\prime}(\alpha)$ and $\tau(\gamma)=\sigma(\alpha)$; hence $\sigma^{\prime}(\alpha) \neq \sigma(\alpha)$. By the choice of $\sigma^{\prime}$, it follows that $\sigma^{\prime}(\alpha) \neq \tau(\delta)$ although $\sigma^{\prime}(\alpha)=\tau^{\prime}(\delta)$, and hence $\tau^{\prime}(\delta) \neq \tau(\delta)$. Again since $\tau^{\prime}$ is covered, it follows that there's a $\beta<|\sigma|$ such that $\tau^{\prime}(\delta)=$ $\sigma^{\prime}(\beta)$ and $\tau(\delta)=\sigma(\beta)$. Hence $\sigma^{\prime} \frown \tau^{\prime}$ is faithful.

Since $\sigma^{\prime}$ is $1-1$, there is a 1-1 mapping $\pi$ defined on the range of $\sigma^{\prime} \frown \tau^{\prime}$ such that $\pi \circ \sigma^{\prime}=1_{|\sigma|}$. Let $\xi=\pi \circ \tau^{\prime}$; then $\imath \frown \xi$ is a faithful extension of 1 such that $\bar{X} \mathrm{l} \Rightarrow_{L} \bar{A} \xi$. By Proposition 13, it follows that $\bar{X} \sigma \Rightarrow_{N L} \bar{A} \tau$, as desired.

\section{Concluding remarks}

In $\S \S 3$ and 4 , we considered two analyses of manifest consequence based on the the sketches that Fine gives in his book. Although the two analyses look quite different, it turns out, by Propositions 18 and 19, that they extensionally coincide. Morever, Propositions 8 and 13 show that each of these analyses leads to further equivalences as well. These results testify to the naturalness of the underlying concept of manifest consequence, and also to the naturalness of its generalization, consequence relative to schemes of coordination.

However, it hardly needs to be said that the mathematical naturalness of a concept does not guarantee its viability for some explanatory role. We conclude the paper with two remarks on the role of coordination schemes in the program of semantic relationism. The first remark articulates conceptual connections between relationism and its rivals, Fregeanism and referentialism. The second remark suggests a way to explore the connection between coordination schemes and the phenomena they are purported to explain.

\subsection{COORDINATION AND FREGEANISM}

On Fine's account, it is the distinctive predicament of reasoning under a coordination scheme that explains the cognitive difference between trivial and nontrivial identities. Reasoning from hypotheses under a scheme is just classical reasoning from specially corresponding propositions, namely propositions resulting from coordinated hypotheses when ordinary objects have all given way to corresponding equivalence classes. Thus, coordinated inference requires a command of $L$-consequence over some body of propositions. However, this account seems rather puzzling. For a command of $L$-consequence over some propositions requires a thinker to discern throughout them the recurrence of objectual constituents. But isn't the whole point of the story to avoid some semantic requirement of a capacity to recognize objects as the same again? 
Consider the analogous question whether the recurrence of Fregean senses must be transparent to a speaker. It would seem that if it is asked "is the sense of 'Hesperus' the same as the sense of 'the evening star'?" then it should be open to the Fregean to allow that the speaker not know the answer, perhaps even despite perfect fluency with the expressions 'Hesperus' and 'the evening star', because the kind of recognition demanded by the question is one in which the senses no longer work as modes of presentation but rather appear as objects, presented themselves under varying aspects. In other words, the Fregean may deny that what underpins the cognitive role of modes of presentation is the speaker's recognition of their identity and distinctness as objects. Similarly, in the case at hand, a coordination scheme over a class of Russellian propositions may induce a class of secondary propositions in which objectual constituents give way to representational proxies. But, it would be a mistake to think that these proxies are some special objects that are as it were intrinsically naked. Nor do they so happen to reveal their identities to the speaker like travelers before a customs agent. Rather, the identity of the proxies is constitued by the requirements which the grasp of meaning imposes. After all, they are equivalence classes of occurrences, induced by the coordination scheme whose extension the grasp of meaning compels the speaker to acknowledge. Or, in Copernican: it is not that understanding conforms to the identity of the proxies, but that the proxies conform themselves to the understanding. ${ }^{43,44}$

In this way, the analysis of Footnote 14 presupposes that a grasp of the meaning of sentences would guarantee the existence of a class of entities whose recurrence, at least throughout the propositional contexts induced by those sentences, must be transparent to the speaker. If we consider only coordination schemes which are sound, there is a many-one correspondence of these proxy elements to objectual constituents, such that distinct proxy elements correspond to the same object precisely when, as far as a perfect grasp of meaning can go, it is an open question whether the object corresponding to the one proxy is the same as the object that corresponds to the other. A referentialist might be troubled by this introduction of proxy elements and of a second layer of content to contain them. ${ }^{45}$

The basic question at issue here is whether or not there exist entities corresponding to the occurrences of names, such that

(1) the correspondence of the entity to an occurrence is a semantic property of the occurrence,

(2) this correspondence is an intrinsic property of the occurrence, ${ }^{46}$

(3) different such entities correspond to different occurrences iff the occurrences do not corefer transparently, and 
(4) the transparency of recurrence of the entities corresponding to occurrences of names is presupposed in the full understanding of sentences containing those occurrences.

According to semantic relationism, there are entities satisfying $(1,3,4)$, but there are no entities satisfying $(1,2,3,4)$ or even just $(1,2,3) .{ }^{47}$ An "intrinsicalist" will hold that (1) implies (2), whereas a relationist rejects this entailment. Fine's referentialist will hold that (1) excludes (3). ${ }^{48}$ Fine's 'Fregean' takes the notion of sense to satisfy all of $(1,2,3,4) .{ }^{49}$

At this point it is surely worth recapitulating the contrasts that Fine draws between the relationist and 'Fregean' viewpoints. ${ }^{50}$ The relationist interpretation of a sentence in a context assigns to it as propositional content a Russellian proposition together with a coordination scheme. The resulting coordinated proposition determines precisely the same function from possible worlds to truth-values as the underlying Russellian proposition. Hence, the content assigned by the relationist and the content assigned by the referentialist have the same modal profile. Furthermore, the relationist does not introduce into propositional content anything like a qualitative specification of objects designated, and so avoids the epistemological quandaries of the 'Fregean'. Rather, might be hoped that what is required to understand a sentence as expressing a coordinated proposition would epistemically transcend what is required to understand a sentence as expressing a Russellian proposition only by carrying with it a recognition of those truths of identity which hold in virtue of meaning.

\subsection{OCCURRENCES}

The fundamental notion of this paper is probably that of an occurrence. In some respects, the present treatment has been rather naïve. For example, some basic results of this paper appear to have an underlying "algebraic" character which is obscured by set-theoretic concreteness. It might be well worth seeking an appropriate abstract axiomatization of occurrencehood. ${ }^{51}$ Moreover, we have simply assumed a natural wellordering of the hypotheses of an inference, allowing an ordinal specification of the positions of occurrences. But in real life, the structures of occurrence are richer. ${ }^{52}$ Consider, for example, the history of Russell's famous article "On Denoting". Russell's article was produced over a weekend as a stack of marked-up sheets of paper. Seen from an alien perspective, ${ }^{53}$ it looks as though the marked-up sheets carried with them a signal that hit the printer's office and burst out in several thousand directions, gripping the collective imagination of philosophers throughout the English-speaking world. Each new bearer of the signal carries with it some of the propagating power of the original, so it too is subject to reprinting, as well as to transcription, quotation, and so on. Thus, it looks like there corresponds to "On Denoting" some complicated branching process. Perhaps 
the process should be distinguished from the paper, since the process and the paper may be differently individuated. It might be more plausible to identify the paper with the signal transmitted. In any case, the question now arises how, within even just this fragment of a very rich ontology, we should go about locating the occurrences of names like "Scott" in the sentences, or of men like Scott in the propositional content. Inasmuch as the content of the paper should be identified with what Russell actually said, then it might be most natural to identify occurrences of "Scott", the name, with occurrences in the signal, and the underlying coordinated occurrences of Scott, the man, with occurrences in the signal's content. But an account of the cognitive value for an audience of Russell's pronouncements may require attention to the details of propagation, and therefore require us to identify occurrences relative to some bearer of the signal, for example when the Gray's Elegy passage is hashed out in a seminar. Beneath the manner of realization of sentences, there would correspond a structure over propositional contents, and a coordination scheme that pervades the structure. Perhaps it is safe to conclude that the picture invites more scrutiny. ${ }^{54}$

\section{Notes}

${ }^{1}$ Fine (2007, henceforth $S R$ ), 3, 35.

${ }^{2}$ See for example Wettstein (1986) and Kaplan (1990).

${ }^{3} S R, 43$.

${ }^{4} S R$ 5, 39-40.

${ }^{5} S R, 43$.

${ }^{6}$ Frege (1892).

${ }^{7} S R, 62$.

${ }^{8} S R, 49-50$.

${ }^{9} S R, 48$.

${ }^{10} S R, 48$.

${ }^{11} \mathrm{SR}, 55 \mathrm{ff}, 136 \mathrm{n} 14$.

${ }^{12}$ See Goldfarb (2001) for an illuminating treatment of this point.

${ }^{13}$ Cf. Tarski, A., (1933), reprinted in Corcoran (1983), 200.

${ }^{14}$ At least, not in $Z F$. In $N B G$, they would still be second-class citizens.

15 See for example Carnap (1947), 23ff; Montague (1960), 163, and Lewis (1970), 23.

${ }^{16}$ This was already suggested in Carnap's original presentation (1947), 29, but the approach persists; see Soames (1987) for a thorough discussion.

${ }^{17}$ See Moore (1899), Russell (1903).

${ }^{18}$ Kaplan (1989).

${ }^{19}$ In his later (1986), Kaplan develops in more detail an analogy of a proposition with what he calls a "valuated formula", which is, roughly speaking, a formula together with an assignment of values to its free variables. The latter notion is more or less familiar from logic, as a formula with parameters drawn from a given domain; see for example Krivine (2007), 63. But on Kaplan's approach, as on the approach to be taken in what follows, one and the same expression results from assigning $a$ as the value of ' $x$ ' and ' $y$ ' in the formulas ' $F x$ ' and ' $F y$ '. 
${ }^{20}$ Of course, this presupposes some procedure to ensure that the new terms do not clash with the existing apparatus-for example, that they do not overlap with the set of variables. Cf. Kaplan (1986), 274.

${ }^{21}$ Cf. Kaplan 1989, 541ff, and 1986, 251.

${ }^{22}$ As Kaplan puts it: 'Assigning me to ' $x$ ' yields a valuation of

$[(y) y$ is unmarried $\supset x$ is unmarried $]$

which is not true in the domain of bachelors" (Kaplan 1986, 250).

${ }^{23}$ The evaluation of contents in the earlier (Kaplan 1989, circulated in the 1970s) takes a smaller step to the same result, despite its possibilistic reading of the quantifiers. On this alternative approach, a non-descriptive linguistic term may receive as its content a 'fallback value', $\dagger$, which is excluded from the domain of quantification and from the extensions of predicates. Now, suppose that the term $a$ receives the value $\dagger$. Then, at a world and time where the extension of the predicate $F$ coincides with the domain of quantification, the Kaplanian contents of $\ulcorner\neg F a\urcorner$ and $\ulcorner\exists x \neg F x\urcorner$ are true and false respectively. The evaluation of Kaplanian contents resembles in this respect a special case of evaluation of $\mathcal{L}_{\mathfrak{U}}$, in which the domain of the structure includes all but exactly one of the elements of $\mathcal{U}$.

${ }^{24}$ This is a relettering of the statement at $S R, 48$.

${ }^{25} \mathrm{SR}, 135 \mathrm{n} 11$.

${ }^{26} \mathrm{SR}, 135 \mathrm{n} 11$.

${ }^{27} \mathrm{I}$ 'm indebted to Nathan Salmon for discussion here.

${ }^{28}$ According to the U.S. Department of Justice (2011), "in 2010, 7.0\% of households in the United States... had at least one member age 12 or older who experienced one or more types of identity theft victimization."

${ }^{29}$ This stipulation can be weakened in various ways. As a first pass, the problem actually arises only in case $\operatorname{Card}(\mathcal{U}-\operatorname{Ran}(\sigma \frown \tau))<\operatorname{Card}(|\sigma \frown \tau|)$. This condition holds provided that, for example, $\mathcal{U}$ is the domain of a model of set theory and $\operatorname{Ran}(\sigma \frown \tau)$ exists as a set in that model.

${ }^{30}$ For the sake of informal discussion, $M$-consequence may here be taken to be any consequence relation $I$ over $\mathcal{L}_{\mathfrak{U}}$ such that $M(K \cap L) \subseteq I \subseteq M L$.

${ }^{31} S R, 41$. The arguments of this passage may rely partly on earlier remarks about an "intuitive notion of meaning" with respect to which trivial and nontrivial statements of identity would differ (35).

${ }^{32}$ In Fine's initial presentation ( $S R$ 5) he does not sharply distinguish strict coreference from coordination; but the official exposition ( $S R$ 54ff) draws the distinction carefully.

${ }^{33} \mathrm{SR}, 54 \mathrm{ff}$.

${ }^{34} S R, 52$.

${ }^{35} S R, 67$.

${ }^{36} S R, 73$.

${ }^{37}$ For an example, see again U.S.D.O.J., op. cit.

${ }^{38} \mathrm{SR}, 136 \mathrm{n} 14$.

${ }^{39} \mathrm{SR}, 108 \mathrm{ff}$.

${ }^{40}$ Maybe not all historians of philosophy are fated to benefit from Proposition 12.

${ }^{41}$ In particular, the underlying relation must invalidate statements of distinctness. Otherwise, the proposition $\ulcorner a=b\urcorner K$-entails the absurdity under the scheme which does not coordinate the two occurrences; yet we need to explain how the truth of identity could be an open question.

${ }^{42}$ There seems to be room here for improvements of Proposition 16. Confusion about hypotheses is a fact of life, but we might still want to find consequences. We could ensure the soundness of our inferences by insisting that their soundness be manifest. But is there a better, or even optimal strategy for reasoning from possibly confused hypotheses in such a way as to 
quarantine the confusions, wherever they happen to be? Note that it will not suffice to insist that the conclusion contain none of the objects that had been confused, since inferentially exploited objects can be laundered out through quantification.

${ }^{43}$ I take it that this is the source of immunity of Finean proxies to the fantastical "Bruce" objection $(S R, 36)$.

${ }^{44} \mathrm{I}$ ' $\mathrm{m}$ indebted here to the comments of an anonymous reviewer.

${ }^{45}$ Cf. SR, 57.

${ }^{46}$ More precisely, (2) amounts to the claim that the correspondence derives from those features of the occurrence of the name which are independent of the sentential context of the occurrence. Supposing that, roughly speaking, the occurrence of a name in a sentence, considered independently of the sentence, is just the name, then, (2) may be understood as the claim that the correspondence of the entity to the occurrence derives from the properties of the name which occurs.

${ }^{47}$ To derive the property (1), it suffices to apply to a coordinated proposition the following theorem: for any set $S$ and any equivalence relation $E$ over $S$, there is a function $f$ such $\forall x \in$ $S(f(x)=f(y) \leftrightarrow E x y)$. To derive (4), note that full understanding presupposes grasp of the extension of the coordination scheme, and that the formula just given is manifestly applicable.

${ }^{48} S R, 35$.

${ }^{49}$ With respect to original Sinn, it seems clear that Frege accepted (3) and (4); if, taking a stand on a controversial interpretive issue, we grant that Frege did think of his doctrine of Sinn as part of a semantic theory, then he might also accept (1). However, Frege maintained that (1) excludes (2): see for example Frege (1883), in Austin, trans., (1950), pp. x and 71, and the letter to Russell from 29 June 1902, in McGuinness et al., (1980), 135ff. For representative discussion of these issues, see Potter and Ricketts (2011).

${ }^{50}$ See $S R, 57 \mathrm{ff}$ for Fine's own account of the contrasts.

${ }^{51}$ This suggestion is due to an anonymous reviewer.

${ }^{52}$ Cf. $S R, 124-125$.

${ }^{53}$ I hope that Kaplan would not be offended by this allusion to his (1990).

${ }^{54}$ I wish to thank Roger Clarke, Sid Grewal, Yannig Luthra, Nathan Salmon, and Ori Simchen for discussion. Thanks are due as well to the two anonymous reviewers for this journal, whose generosity enriched the paper throughout. I should note here my primary motive for the explorations of this paper, which is curiosity.

\section{References}

Carnap, R. (1947). Meaning and Necessity. Chicago: University of Chicago Press.

Fine, Kit (2007). Semantic Relationism. Oxford: Blackwell.

Frege, G. (1892). "Über Sinn und Bedeutung”. Reprinted in McGuinness, B., ed. (1984). Gottlob Frege: Collected Papers on Mathematics, Logic and Philosophy. Oxford: Blackwell.

Frege, G. (1883). Die Grundlagen der Arithmetik. Translated in Austin, J. L. (1950). The Foundations of Arithmetic. London: Blackwell.

Goldfarb, W. (2001). "Frege's conception of logic". In Floyd, J., and Shieh, S. (2001). Future Pasts.. New York: Oxford University Press.

Kaplan, D. (1986). “Opacity.” In Hahn, L.E., and Schilpp, P.A., eds. (1998). The Philosophy of W.V. Quine. LaSalle: Open Court.

—. (1989). "Demonstratives.” In Almog, J., Perry, J., and Wettstein, H., eds. (1998). Themes from Kaplan, 481-563. New York: Oxford.

—. (1990). "Words." Proc. Arist. Soc. Supp. Vols., Vol. 64, pp. 93-119.

Krivine, J. L. (2007). Theorie des Ensembles. Paris: Cassini. 
Lewis, D. (1970). "General Semantics”. Synthese, Vol. 22, No. 1-2, 18-67.

McGuiness, B., ed., and Kaal, H., trans. Gottlob Frege: Philosophical and Mathematical Correspondence. Oxford: Blackwell.

Montague, R. (1960). “On the Nature of Certain Philosophical Entities”. The Monist 53, 15994.

Moore, G. E. (1899). "The nature of judgment.” Mind, New Series, Vol. 8, No. 30, 176-193.

Potter, M., and Ricketts, T., eds. (2011). The Cambridge Companion to Frege. New York: Cambridge University Press.

Russell, B. A. E. (1903). The Principles of Mathematics. London: Norton.

Soames, S. (1987). "Direct reference, propositional attitudes, and semantic content." Philosophical Topics 15: 47-87.

Tarski, A. (1933). "The concept of truth in formalized languages". Translated in Corcoran, J., ed. (1983). Logic, Semantics, Metamathematics. Indianapolis: Hackett.

United States Department of Justice. (2011). "Crime data brief: Identity theft reported by households, 2005-2010”. Retrieved 2 January 2013 from

http://bjs.ojp.usdoj.gov/content/pub/pdf/itrh0510.pdf.

Wettstein, H. K. (1986). "Has semantics rested on a mistake?" The Journal of Philosophy, 83 (4):185-209. 